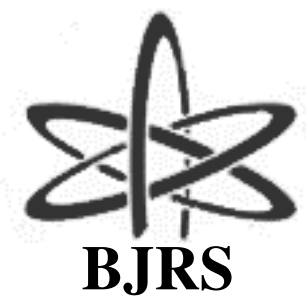

BRAZILIAN JOURNAL

$\mathrm{OF}$

RADIATION SCIENCES

03-1A (2015) 01-14

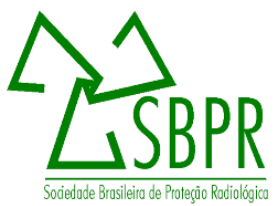

\title{
Verificação do uso de dosímetros individuais em serviço de medicina nuclear de Pernambuco nos anos de 2002 a
}

\section{0}

Renata Farias de Lira ${ }^{a}$, Fernando Roberto de Andrade Lima. ${ }^{\text {a, }}$, José Wilson Vieira ${ }^{\mathrm{c}}$

${ }^{a}$ Departamento de Energia Nuclear - Universidade Federal de Pernambuco - (DEN/UFPE)

Av. Prof. Luiz Freire, 1000, Recife - PE - CEP 50.740-540

renatafariasdelira@hotmail.com

${ }^{b}$ Centro Regional de Ciências Nucleares do Nordeste (CRCN-NE)

Comissão Nacional de Energia Nuclear (CNEN)

Av. Professor Luiz Freire, 200, Recife - PE - CEP 50740-540

falima@cnen.gov.br

${ }^{c}$ Escola Poolitécnica de Pernambuco - UPE

Rua Benfica, 455 - Madalena - Recife/PE CEP: 50720-001

jose.wilson59@uol.com.br

\section{Resumo}

A medicina nuclear $(\mathrm{MN})$ é uma especialidade médica que utiliza material radioativo associado a fármacos para obter imagens diagnósticas e realizar procedimentos terapêuticos. Como qualquer atividade que faça uso de radiação ionizante, a MN deve ser justificada, otimizar seus procedimentos de trabalho e aplicar a limitação de dose. O uso de dosímetro individual é de extrema necessidade para avaliar as doses recebidas pelos trabalhadores e verificar se estas estão de acordo com as normas estabelecidas pela Comissão Nacional de Energia Nuclear (CNEN). Este estudo tem como objetivo verificar o uso de dosímetros pelos indivíduos ocupacionalmente expostos (IOE) em um Serviço de Medicina Nuclear (SMN) de Pernambuco a fim de controlar as exposições ocupacionais. Foram avaliados 56 IOE no período de 2002 a 2010. Verificou-se que o ano de 2005 apresentou maior número de IOE não monitorados, enquanto o ano de 2009 não apresentou IOE não monitorados. Observou-se ainda que com o passar dos anos apesar do aumento do número de trabalhadores a monitoração se mostrou mais efetiva. Não se pode 
afirmar o motivo pelo qual a monitoração se tornou mais efetiva, porém acredita-se que uma maior preocupação com a radioproteção esteja sendo aplicada aos procedimentos de trabalho do SMN avaliado.

Palavras-chave: colocar Serviço de Medicina Nuclear, Dosímetro individual, Exposição ocupacional.

\section{INTRODUÇÃO}

A Medicina Nuclear (MN) é uma especialidade médica que utiliza radionuclídeos com características físicas e químicas adequadas, tanto para fins diagnósticos como terapêuticos. É capaz de evidenciar bem a função do órgão em estudo, enquanto a radiologia convencional, o ultrassom, a tomografia computadorizada e a ressonância magnética destacam melhor os aspectos morfológicos e anatômicos. [1]

O princípio básico da MN consiste em avaliar a função de um determinado órgão utilizando uma substância marcada com radionuclídeos que seja, normalmente, captada ou metabolizada pelo órgão que se deseja estudar [1]. Essas substâncias são denominadas radiofármacos e as características físico-químicas do fármaco, determinam sua fixação no órgão ou tecido alvo e sua eliminação pelo organismo, enquanto as características físicas do radionuclídeo determinam a aplicação do composto em diagnóstico ou terapia. [2]

Toda atividade envolvendo aplicação de radiação ionizante, deve ser justificada e ter seus procedimentos de trabalho otimizados, assim como deve respeitar a limitação de dose, de acordo com os três princípios básicos da proteção radiológica: justificação, limitação de dose e otimização.

O objetivo primário da proteção radiológica é fornecer um padrão apropriado de proteção para o homem, sem limitar os benefícios criados pela aplicação das radiações ionizantes. [3]

O controle de trabalhadores de áreas controladas deve ser executado através de monitoração individual, avaliação de doses e supervisão médica. A monitoração individual é a monitoração de pessoas por meio de dosímetros individuais colocados sobre o corpo [4]. Os dosímetros são instrumentos que indicam a exposição ou a dose absorvida a que um indivíduo foi submetida.

A utilização do dosímetro é necessária para obter uma estimativa da dose efetiva e/ou da dose equivalente no cristalino e extremidades, compatível com a atividade exercida, de modo a demonstrar conformidade com os requisitos administrativos e operacionais estabelecidos pelo serviço, bem como contribuir para o controle e melhoria da operação da instalação. [5] 


\section{MATERIAIS E MÉTODOS}

Este trabalho avaliou o uso de dosímetros individuais de 56 trabalhadores do Serviço de Medicina Nuclear (SMN) do Hospital das Clínicas de Pernambuco (HC/UFPE) no período do ano de 2002 ao ano de 2010.

Foi realizado o levantamento do registro de monitoração do serviço, avaliando, os meses que não houve monitoração e a quantidade de IOE que não fizeram uso do dosímetro por mês e por ano.

Foram analisados nos registros cada mês de cada ano do estudo as doses recebidas pelos trabalhadores e observando quantos e quais trabalhadores não utilizaram o dosímetro individual.

\section{RESULTADOS E DISCUSSÕES}

Os resultados da análise dos registros da instalação são apresentados nas Tabelas 1 a 9, que mostram o ano do estudo divididos em meses, também são vistos nas tabelas os meses em que não houve monitoração, a quantidade de IOE não monitorados por mês e por ano, a quantidade total de trabalhadores na instalação a cada ano. 
Tabela 1. Verificação do uso de dosímetro individual no SMN - HC/UFPE em 2002

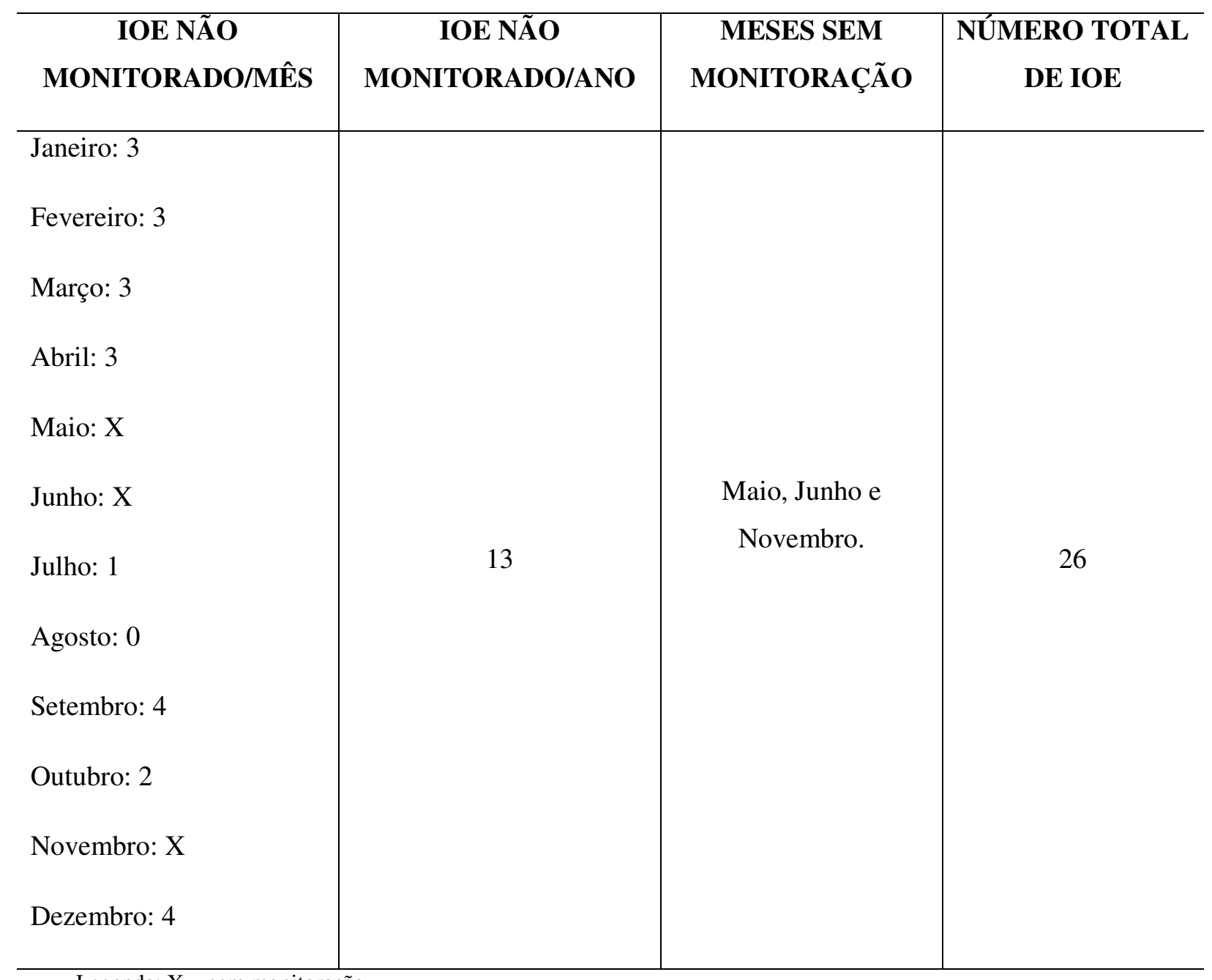

Legenda: $\mathrm{X}=$ sem monitoração

Vê-se na Tabela 1que dos 26 IOE monitorados no ano de 2002, 13 não utilizaram o dosímetro no decorrer do ano. Observa-se também que os meses de maio, junho e novembro foram os meses em que algum dos IOE não forma monitorados. Ainda é visto que os meses de setembro e dezembro foram os que mais tiveram trabalhadores não monitorados. 
Tabela 2. Verificação do uso de dosímetro individual no SMN - HC/UFPE em 2003

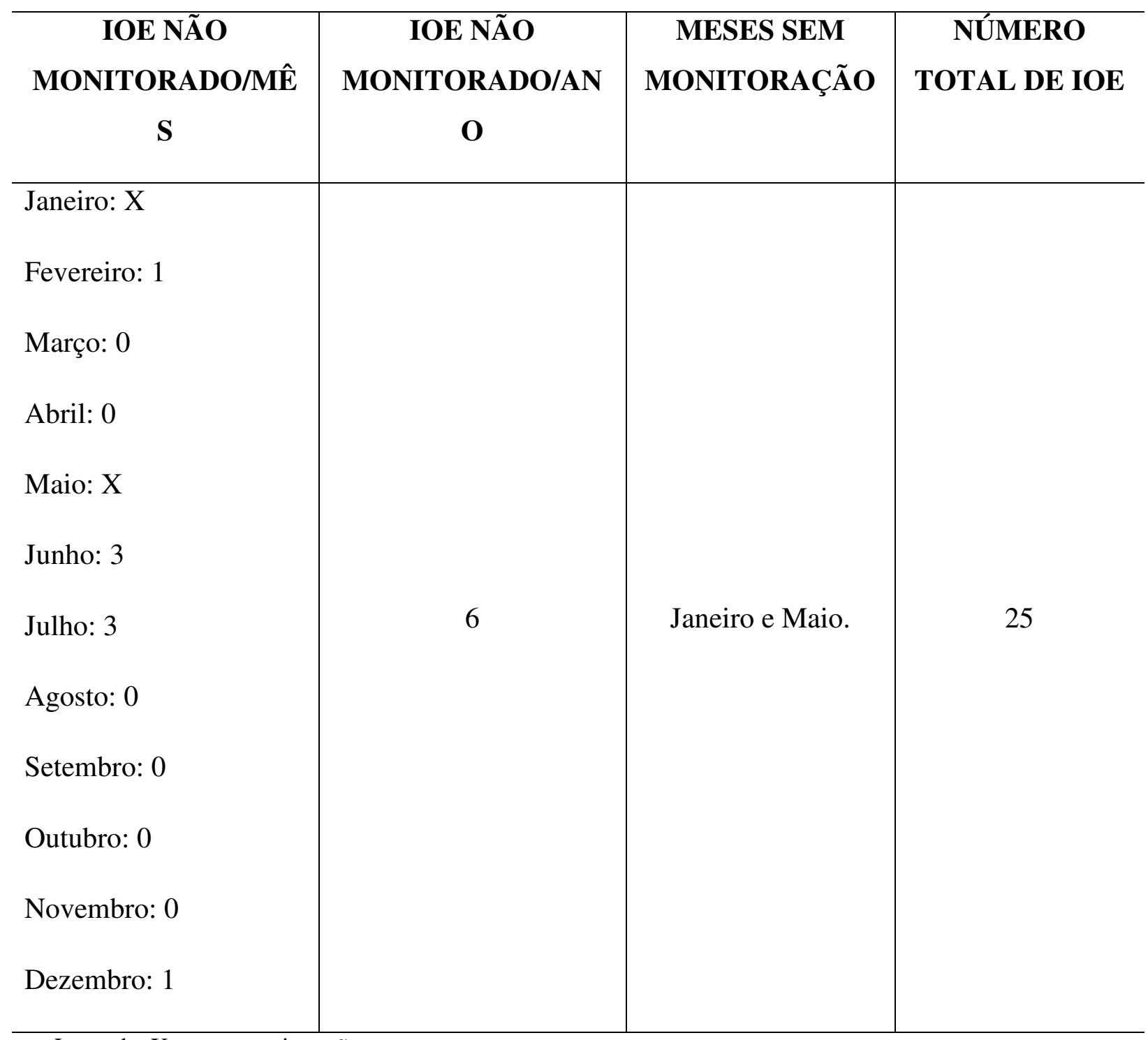

Legenda: $\mathrm{X}=$ sem monitoração

Na Tabela 2, observa-se que 25 IOE foram analisados, sendo 6 destes não monitorados no decorrer do ano de 2003. Vê-se ainda que os meses de junho e julho foram os meses com mais funcionários não monitorados, bem como, que os meses de janeiro e maio não houve monitoração. 
Tabela 3. Verificação do uso de dosímetro individual no SMN - HC/UFPE em 2004

\begin{tabular}{cccc}
\hline IOE NÃO & IOE NÃO & MESES SEM & NÚMERO \\
MONITORADO/MÊ & MONITORADO/AN & MONITORAÇÃO & TOTAL DE IOE \\
S & O & &
\end{tabular}

Janeiro: 0

Fevereiro: 0

Março: 2

Abril: 0

Maio: 0

Junho: 3

Julho: 0

Outubro.

30

Agosto: 0

Setembro: 6

Outubro: X

Novembro: 4

Dezembro: 1

Legenda: $\mathrm{X}=$ sem monitoração

A Tabela 3 apresenta os dados de 30 trabalhadores que foram observados durante o ano de 2004, sendo 12 deles não monitorados no decorrer do ano. A tabela ainda apresenta o mês de outubro como único mês sem monitoração e o mês de setembro como mês com mais IOE não monitorado no ano do estudo. 
Tabela 4. Verificação do uso de dosímetro individual no SMN - HC/UFPE em 2005

\begin{tabular}{cccc}
\hline IOE NÃO & IOE NÃO & MESES SEM & NÚMERO \\
MONITORADO/MÊS & MONITORADO/ANO & MONITORAÇÃO & TOTAL DE IOE
\end{tabular}

Janeiro: 1

Fevereiro: 2

Março: 0

Abril: 1

Maio: 1

Junho: 1

Julho: 0

9

Nenhum.

38

Agosto: 0

Setembro: 2

Outubro: 1

Novembro: 2

Dezembro: 0

\footnotetext{
Legenda: $\mathrm{X}=$ sem monitoração
}

A Tabela 4 mostra que não houve nenhum mês sem monitoração, e apenas 9 trabalhadores não monitorados dos 38 avaliados. Ainda mostra que os meses de fevereiro, setembro e novembro foram os meses com mais IOE não monitorados. 
Tabela 5. Verificação do uso do dosímetro individual no SMN - HC/UFPE em 2006

\begin{tabular}{cccc}
\hline IOE NÃO & IOE NÃO & MESES SEM & NÚMERO \\
MONITORADO/MÊ & MONITORADO/AN & MONITORAÇÃO & TOTAL DE IOE \\
S & O & &
\end{tabular}

Janeiro: 0

Fevereiro: $\mathrm{X}$

Março: 2

Abril: 1

Maio: 7

Junho: 0

Fevereiro, Junho e

Julho: 7

Dezembro.

Agosto: 0

Setembro: 8

Outubro: 1

Novembro

Dezembro

Legenda: $X$ = sem monitoração

Na Tabela 5 são vistos os dados de 37 funcionários, sendo 22 deles não monitorados no decorrer do ano. A tabela mostra também que os meses de fevereiro, junho e dezembro não tiveram monitoração e ainda o mês de setembro como o maior número de trabalhadores não monitorados. 
Tabela 6. Verificação do uso do dosímetro individual no SMN - HC/UFPE em 2007

\begin{tabular}{cccc}
\hline IOE NÃO & IOE NÃO & MESES SEM & NÚMERO \\
MONITORADO/MÊ & MONITORADO/AN & MONITORAÇÃO & TOTAL DE IOE \\
S & O & &
\end{tabular}

Janeiro: 1

Fevereiro: 1

Março: 1

Abril: 3

Maio: 0

Junho: 0

Julho: 0

8

Nenhum.

27

Agosto: 2

Setembro: 3

Outubro: 0

Novembro: 1

Dezembro: 1

Legenda: $X$ = sem monitoração

Os dados apresentados na Tabela 6 são referentes a 27 IOE que foram avaliados no ano de 2007, destes, 8 não tiveram monitoração individual. Ainda vê-se na tabela que nenhum mês desse ano ficou sem monitoração, enquanto os meses de abril e setembro apresentaram a maior quantidade de funcionários que não fizeram uso do dosímetro pessoal. 
Tabela 7. Verificação do uso do dosímetro individual no SMN - HC/UFPE em 2008

\begin{tabular}{cccc}
\hline IOE NÃO & IOE NÃO & MESES SEM & NÚMERO \\
MONITORADO/MÊS & MONITORADO/ANO & MONITORAÇÃO & TOTAL DE IOE
\end{tabular}

Janeiro: 0

Fevereiro: 0

Março: 0

Abril: 3

Maio: 1

Junho: 0

Julho: 0

$6 \quad$ Nenhum.

29

Agosto: 1

Setembro: 1

Outubro: 0

Novembro: 2

Dezembro: 0

Legenda: $\mathrm{X}=$ sem monitoração

A Tabela 7 exibe o mês de abril como o com maior quantia de IOE não monitorado e que não houve um mês sem monitoração. Ainda é mostrado na tabela que 6 dos 29 trabalhadores analisados não utilizaram dosímetro individual durante o ano de 2008. 
Tabela 8. . Verificação do uso do dosímetro individual no SMN - HC/UFPE em 2009

\begin{tabular}{cccc}
\hline IOE NÃO & IOE NÃO & MESES SEM & NÚMERO \\
MONITORADO/MÊS & MONITORADO/ANO & MONITORAÇÃO & TOTAL DE IOE
\end{tabular}

Janeiro: X

Fevereiro: 0

Março: 0

Abril: 0

Maio: 0

Junho: 0

Janeiro, Julho,

Julho: X

0

Agosto e Dezembro.

47

Agosto: X

Setembro: 0

Outubro: 0

Novembro: 0

Dezembro: X

Legenda: $X$ = sem monitoração

A Tabela 8 representa o ano com maior número de meses sem monitoração, sendo eles janeiro, julho, agosto e dezembro. Por outro lado, a tabela mostra não houve IOE sem monitoração dos 47 analisados. 
Tabela 9. Verificação do uso do dosímetro individual no SMN - HC/UFPE em 2010

\begin{tabular}{cccc}
\hline IOE NÃO & IOE NÃO & MESES SEM & NÚMERO \\
MONITORADO/MÊS & MONITORADO/ANO & MONITORAÇÃO & TOTAL DE IOE
\end{tabular}

Janeiro: 5

Fevereiro: 0

Março: 0

Abril: 0

Maio: 0

Junho: X

Junho e Dezembro.

Julho: 1

6

56

Agosto: 0

Setembro: 0

Outubro: 0

Novembro: 0

Dezembro: $\mathrm{X}$

Legenda: $\mathrm{X}=$ sem monitoração

Na Tabela 9 são mostrados 6 trabalhadores não monitorados dos 56 avaliados no ano de 2010 . Ainda é visto na tabela que nos meses de junho e dezembro não houve monitoração e também que o mês de janeiro teve 5 dos 6 IOE não monitorados.

Após a análise dos dados apresentados na tabela pode-se observar que à medida que os anos se passaram a quantidade de IOE do SMN aumentou, acredita-se que devido ao crescimento da realização de exames. Mesmo com uma maior quantidade de trabalhadores, o número de funcionários não monitorados não cresceu, levando a crer que os princípios da proteção radiológica vêm sendo aplicados adequadamente e/ou que a fiscalização tem sido mais efetiva.

Nota-se ainda que o ano de 2009 não apresentou IOE não monitorados e apresentou o segundo maior número de trabalhadores do estudo. Percebe-se também que o ano de 2006 foi o ano que 
apresentou a maior quantidade de funcionários não monitorados, sendo 22 dos 37 monitorados. Pode-se ver que após 2006 houve a diminuição dos IOE não monitorados, talvez pelo cumprimento mais fiel dos padrões de segurança.

Não se pode afirmar o real motivo do aumento de trabalhadores, mas acredita-se que popularização da $\mathrm{MN}$ tornou o serviço mais atuante e por isso a necessidade de mais trabalhadores. Também não há como afirmar o motivo da maior eficácia da monitoração dos funcionários, porém um provável motivo é a maior fiscalização da CNEN quanto à proteção radiológica, assim como maior conscientização dos IOE relacionada à necessidade de atender aos procedimentos de proteção e segurança.

Para manutenção de uma boa monitoração individual, sugere-se a aplicação de cursos e treinamentos periodicamente para os IOE da instalação, a fim de aprimorar os procedimentos de trabalho e gerar um maior grau de conscientização da segurança, tanto do próprio trabalhador, como dos companheiros de trabalho e do público em geral.

\section{CONCLUSÕES}

De acordo com os resultados obtidos e apresentados, pode-se concluir que:

- Em apenas um ano do estudo não houve IOE sem monitoração;

- O trabalho apresentou que em todos os anos analisados, teve ao menos um mês sem monitoração;

- A quantidade de trabalhadores do SMN aumentou com o passar dos anos;

- Para uma monitoração mais eficaz, faz-se necessário a conscientização dos trabalhadores quanto ao uso dos dosímetros individuais.

\section{AGRADECIMENTO}

Agradecimentos à CNPq pelo consentimento da bolsa de pós-graduação, ao Grupo de Dosimetria Numérica (GDN) e ao CRCN/NE pelo apoio e permissão do uso de suas instalações para desenvolvimento do trabalho e ao HC/PE pela concessão dos dados utilizados no estudo. 


\section{REFERÊNCIAS}

[1] MESQUiTA, E. T, MESQUITA, C. T, FONSECA, L. M.-Medicina Nuclear Aplicada a Cardiologia, Editora Atheneu, Volume 4, 2001.

[2] OLIVEIRA, R.. SANTOS, D. FERREIRA, D. COELHO, P. VEIGA, F.. “Preparações radiofarmacêuticas e suas aplicações". Revista Brasileira de Ciências Farmacêuticas. abr/jun Vol. 42, n. ${ }^{\circ} 2,2006$.

[3] COMMISSION OF THE EUROPEAN COMMUNITIES (CEC). Alara from theory towards practice, Final Report, 1991.

[4] COMISSÃO NACIONAL DE ENERGIA NUCLEAR. CNEN-NN 3.01 - Diretrizes básicas de Proteção Radiológica, 2011.

[5] MINISTÉRIO DA SAÚDE - PORTARIA DA SECRETARIA DE VIGILÂNCIA SANITÁRIA No 453 - Diretrizes de Proteção Radiológica em Radiodiagnóstico Médico e Odontológico, 1998. 